\title{
Reporting Biopsy Margin Status for Cutaneous Basal Cell Carcinoma: To Do or Not to Do
}

\author{
Muhammad N. Mahmood, MD
}

To the Editor:

In an interesting analysis, Brady and Hossler ${ }^{1}$ (Cutis. 2020;106:315-317) highlighted the limitations of histopathologic biopsy margin evaluation for cutaneous basal cell carcinoma (BCC). Taking into consideration the high prevalence of BCC and its medical and economic impact on the health care system, the issue raised by the authors is an important one. They proposed that pathologists may omit reporting margins or clarify the limitations in their reports. It is a valid suggestion; however, in practice, margin evaluation is not always a simple process and is influenced by a number of factors.

The subject of optimum margins for BCC has been debated over decades now; however, ambiguity and lack of definitive guidelines on certain aspects still remain, leading to a lack of standardization and variability in reporting, which opens potential for error. In anatomical pathology, the biopsies for malignancies are interpreted to confirm diagnosis and perform risk assessment, with evaluation of margins generally reserved for subsequent definitive resections. Typically, margins are not required by clinicians or reported by pathologists in common endoscopic (eg, stomach, colon) or needle core (eg, prostate, breast) biopsies. Skin holds a rather unique position in which margin evaluation is not just limited to excisions. With the exception of samples generated from electrodesiccation and curettage, it is common practice by some laboratories to report margins on most specimens of cutaneous malignancies.

In simple terms, when margins are labeled negative there should be no residual disease, and when they are deemed positive there should be disease still persisting in the patient. Margin evaluation for BCC on biopsies falls short on both fronts. In one analysis, 24\% (34/143) of shave biopsies reported with negative margins displayed residual BCC in ensuing re-excisions (negative predictive value: $76 \%)^{2}$ Standard bread-loafing, en-face margins and inking for orientation utilized to provide a thorough margin evaluation of excisions cannot be optimally achieved on small skin biopsies. Microscopic sections for analysis are 2-dimensional representations of 3-dimensional structures. Slides prepared can miss deeply embedded outermost margins, positioned parallel to the plane of sectioning, thereby creating blind spots where margins cannot be precisely assessed and generating an inherent limitation in evaluation. Exhaustive deeper levels done routinely can address this issue to a certain degree; however, it can be an impractical solution with cost implications and delay in turnaround time.

Conversely, it also is common to encounter absence of residual $\mathrm{BCC}$ in re-excisions in which the original biopsy margins were labeled positive. In one analysis, $49 \%$ of BCC patients $(n=100)$ with positive biopsy margins did not display residual neoplasm on following re-excisions. ${ }^{3}$ Localized biopsy site immune response as a cause of postbiopsy regression of residual tumor has been hypothesized to produce this phenomenon. Moreover, initial biopsies may eliminate the majority of the tumor with only minimal disease persisting. Re-excisions submitted in toto allow for a systematic examination; however, areas in between sections still remain where minute residual tumor may hide. Searching for such occult foci generally is not aggressively pursued via deeper levels unless the margins of re-excision are in question.

Superficial-type BCC (or superficial multifocal BCC) is a major factor in precluding precise biopsy margin evaluation. In a study where initial biopsies reported with negative margins displayed residual BCC in subsequent re-excisions, 91\% (31/34) of residual BCCs were of superficial variety. ${ }^{2}$ Clinically, superficial BCC frequently

From the Department of Laboratory Medicine and Pathology, University of Alberta Hospital, Edmonton, Canada.

The author reports no conflict of interest.

Correspondence: Muhammad N. Mahmood, MD, University of Alberta Hospital, Department of Laboratory Medicine and Pathology, 5B4.55 WMC, 8440-112 St, Edmonton, AB T6G 2B7, Canada (noshpath97@gmail.com).

doi: $10.12788 /$ cutis.0276 
has indistinct borders with subtle subclinical peripheral progression. It has a tendency to expand radially, with the clinical appearance deceptively smaller than its true extent. In a plane of histopathologic section, superficial BCC may exhibit skip zones within the epidermis. Even though the margin may seem uninvolved on the slide, a noncontiguous focus may still emerge beyond the"negative" margin. Because superficial pattern is not unusual as one of the components of mixed histology (composite) $\mathrm{BCC}$, this issue is not just limited to tumors specifically designated as superficial type. ${ }^{4}$

The intent of a procedure is important to recognize. If a biopsy is done with the intention of diagnosis only, the pathologic assessment can be limited to tumor identification and core data elements, with margin evaluation reserved for excisions done with therapeutic intent. However, the intent is not always clear, which adds to ambiguity on when to report margins. It is not uncommon to find saucerization shaves or large punch biopsies for BCC carried out with a therapeutic intent. The status of margin is desired in such samples; however, the intent is not always clearly communicated on requisitions. To avoid any gaps in communication, some pathologists may err on the side of caution and start routinely reporting margins on biopsies.

Taking into account the inaccuracy of margin assessment in biopsies, an argument for omitting margin reporting is plausible. Although dermatologists are the major contributors of skin samples, pathology laboratories cater to a broader clientele. Other physicians from different surgical and medical specialities also perform skin biopsies, and catering to a variety of specialities adds another layer of complexity. A dermatologist may appreciate the debate regarding reliability of margins; however, a physician from another speciality who is not as familiar with the diseases of the integument may lack proper understanding. Omitting margin reporting may lead to misinterpretations or false assumptions, such as, "The margins must be uninvolved, otherwise the pathologist would have said something." This also can generate additional phone or email inquiries and second review requests. Rather than completely omitting them, another strategy can be to report margins in more quantitative terms. One reporting approach is to have 3 categories of involved, uninvolved, and uninvolved but close for margins less than $1 \mathrm{~mm}$. The cases in the third category may require greater scrutiny by deeper levels or an added caveat in the comment addressing the limitation. If the status of margins is not reported due to a certain reason, a short comment can be added to explain the reason.

In sum, clinicians should recognize that "margin negative" on skin biopsy does not always equate to "completely excised." Margin status on biopsies is a data item that essentially provides a probability of margin clearance. Completely omitting the margin status on all biopsies may not be the most prudent approach; however, improved guidelines and modifications to enhance the reporting are definitely required.

\section{REFERENCES}

1. Brady MC, Hossler EW. Reliability of biopsy margin status for basal cell carcinoma: a retrospective study. Cutis. 2020;106:315-317.

2. Willardson HB, Lombardo J, Raines M, et al. Predictive value of basal cell carcinoma biopsies with negative margins: a retrospective cohort study. J Am Acad Dermatol. 2018;79:42-46.

3. Yuan Y, Duff ML, Sammons DL, et al. Retrospective chart review of skin cancer presence in the wide excisions. World J Clin Cases. 2014;2:52-56.

4. Cohen PR, Schulze KE, Nelson BR. Basal cell carcinoma with mixed histology: a possible pathogenesis for recurrent skin cancer. Dermatol Surg. 2006;32:542-551.

\section{Authors' Response}

We appreciate the thorough and thoughtful comments in the Letter to the Editor. We agree with the author's assertion that negative margins on skin specimens does not equate to "completely excised" and that the intent of the clinician is not always clear, even when the pathologist has ready access to the clinician's notes, as was the case for the majority of specimens included in our study.

There is already variability in how pathologists report margins, including the specific verbiage used, at least for melanocytic lesions. ${ }^{1}$ The choice of whether or not to report margins and the meaning of those margins is complex due to the uncertainty inherent in margin assessment. Quantifying this uncertainty was the main reason for our study. Ultimately, the pathologist's decision on whether and how to report margins should be focused on improving patient outcomes. There are benefits and drawbacks to all approaches, and our goal is to provide more information for clinicians and pathologists so that they may better care for their patients. Understanding the limitations of margins on submitted skin specimenswhether margins are reported or not-can only serve to guide improve clinical decision-making.

We also agree that the breadth of specialties of submitting clinicians make reporting of margins difficult, and there is likely similar breadth in their understanding of the nuances of margin assessment and reports. The solution to this problem is adequate education regarding the limitations of a pathology report, and specifically what is meant when margins are (or are not) reported on skin specimens. How to best educate the myriad clinicians who submit biopsies is, of course, the ultimate challenge.

We hope that our study adds information to this ongoing debate regarding margin status reporting, and we appreciate the discussion points raised by the author.

\section{Eric Hossler, MD; Mary Brady, MD}

From the Department of Dermatology, Geisinger Health System, Danville, Pennsylvania.

The authors report no conflict of interest.

\section{REFERENCE}

1. Sellheyer K, Bergfeld WF, Stewart E, et al. Evaluation of surgical margins in melanocytic lesions: a survey among 152 dermatopathologists. J Cutan Pathol. 2005;32:293-299. 